The Astrophysical Journal, 653: L21-L23, 2006 December 10

(C) 2006. The American Astronomical Society. All rights reserved. Printed in U.S.A.

\title{
THE NOT SO EXTRAORDINARY GLOBULAR CLUSTER 037-B327 IN M31¹
}

\author{
Judith G. COHEN ${ }^{2}$ \\ Received 2006 September 25; accepted 2006 October 24; published 2006 November 21
}

\begin{abstract}
A velocity dispersion has been measured for the luminous globular cluster M31 037-B327, claimed to be the most massive star cluster in the Local Group and to be a young "super star cluster" that has survived to an old age. M31 037-B327 has a mass comparable to that of M31 G1 but not significantly larger. Although near the upper end for the mass distribution of globular clusters, it is not an unprecedented extraordinary object.

Subject headings: galaxies: individual (M31) — galaxies: star clusters
\end{abstract}

\section{INTRODUCTION}

The globular cluster 037-B327 is an extremely red nonstellar object close to the disk of M31. Barmby et al. (2002b) calculated its reddening, using photometry in the compiled catalog of M31 globular clusters of Barmby et al. (2000) to be $E(B-V)=1.30 \pm 0.04 \mathrm{mag}$, and from this inferred that it was extremely luminous, with $M_{V}=-11.7$ mag, making it the most luminous globular cluster in M31. The recent compilation of data for M31 globular clusters by the Bologna group (Galleti et al. 2004) also confirms the unusual properties of this object.

Recently, Ma et al. (2006a) have studied this cluster in more detail. They compare their new multicolor photometry to theoretical spectral energy distributions of varying ages to determine the reddening and age of this cluster. They find that it has a photometric mass of $(3.0 \pm 0.5) \times 10^{7} M_{\odot}$; they then claim this object to be the most massive star cluster of any age in the Local Group. This claim is based in part on data from the compiled catalogs of Barmby et al. (2000) for the M31 system and of Harris (1996) for the Milky Way globular cluster system. Ma et al. (2006a) predict that 037-B327 has a onedimensional velocity dispersion of $72 \pm 13 \mathrm{~km} \mathrm{~s}^{-1}$, far larger than that of any other known M31 globular cluster. Velocity dispersions for a number of the brightest globular clusters in M31 were determined by Djorgovski et al. (1997); the highest value they measured was $25.1 \pm 0.3 \mathrm{~km} \mathrm{~s}^{-1}$ for $\mathrm{G} 1$.

\section{2. $\sigma_{v}$ FOR M31 037-B327}

We have obtained high spectral resolution spectra with High Resolution Echelle Spectrometer (HIRES) at Keck (Vogt et al. 1994) of 037-B327 and of G1 in M31, as well as of several metal-rich giants on the upper red giant branch of the Draco dwarf spheroidal galaxy that served as template stars. We took two $1500 \mathrm{~s}$ exposures of the object of interest, one $600 \mathrm{~s}$ exposure of the much brighter (at $V$ ) object G1, and several suitable spectra of template red giants from the same run in early 2006 September. All these spectra were taken with a 1 ."1 $\times$ $7^{\prime \prime}$ slit, giving a spectral resolution of 35,000 with 5 pixels per spectral resolution element $\left(\sim 1.3 \mathrm{~km} \mathrm{~s}^{-1}\right.$ pixel $\left.^{-1}\right)$.

We chose for analysis parts of three different echelle orders with strong broadened spectral features visible in the spectrum of 037-B327. Since the flux for this object was dropping rapidly toward the blue, but the number of strong lines was dropping

\footnotetext{
${ }^{1}$ Based on observations obtained at the W. M. Keck Observatory, which is operated jointly by the California Institute of Technology, the University of California, and the National Aeronautics and Space Administration.

${ }^{2}$ Palomar Observatory, Mail Stop 105-24, California Institute of Technology, Pasadena, CA 91125.
}

rapidly toward the red, this was something of a compromise. Cutouts of the spectra in one of the regions analyzed, that near $\mathrm{H} \alpha$, are shown for the two M31 globular clusters and one of the template objects in Figure 1. It is immediately apparent that $\sigma_{v}$ for 037-B327 is comparable to that of G1 but is not significantly larger. This impression is sustained when one examines the strongest lines in these spectra over their full useful wavelength range, which for the object of interest is from about 5100 to $8350 \AA$.

We used the Fourier quotient method of Sargent et al. (1977) to determine $\sigma_{v}$. We subsequently applied an aperture correction factor of 1.14 following Djorgovski et al. (1997). The resulting $\sigma_{v}$ are given in Table 1 . Our measured $\sigma_{v}$ for G1 is in good agreement with that of Djorgovski et al. (1997) $25.1 \pm 0.3 \mathrm{~km} \mathrm{~s}^{-1}$.

\section{COMPARISON OF M31 037-B327 WITH M31 G1}

G1, if one ignores 037-B327, is widely believed to be the most luminous globular cluster in M31 (see, e.g., Meylan et al. 2001). Gebhardt et al. (2003) suggested, based on Hubble Space Telescope (HST) Space Telescope Imaging Spectrograph spectroscopy, that it contains a $20,000 M_{\odot}$ central black hole. Baumgardt et al. (2003) dispute this; they obtain a good fit to all available data for $\mathrm{G} 1$ with their dynamical model, which does not include an intermediate-mass central black hole.

Ma et al. (2006b) utilize an Advanced Camera for Surveys (ACS) image from HST to determine the structural parameters of 037-B327, and discuss the possibility, suggested earlier by Barmby et al. (2002b) that this object is the nucleus of a dwarf galaxy accreted by M31. The ACS image reveals a dust lane crossing the face of the cluster. Toy models of a uniform extinction over most of the cluster, with much larger $E(B-V)$ over a smaller part of the cluster, suggest that substantial errors in the values of the dereddened fluxes can occur in such circumstances if a standard extinction curve as a function of wavelength is applied assuming a constant $E(B-V)$. The reddening of $\mathrm{G} 1$ is small as it has a projected distance from the center of M31 of $40 \mathrm{kpc}$; we assume only the foreground Galactic reddening applies.

We compare the properties of 037-B327 with those of G1. The values of $\sigma_{v}$ are comparable (see Table 1), with that of G1 perhaps being slightly larger. Since the reddening of 037-B327 is large and spatially variable across the face of the cluster, we prefer to compare the luminosities at $K$ where the impact of the reddening is minimized. We assume both objects are old stellar clusters. This comparison is sure to be more reliable than a similar one making the same assumption carried out at $V$ by Ma et al. 


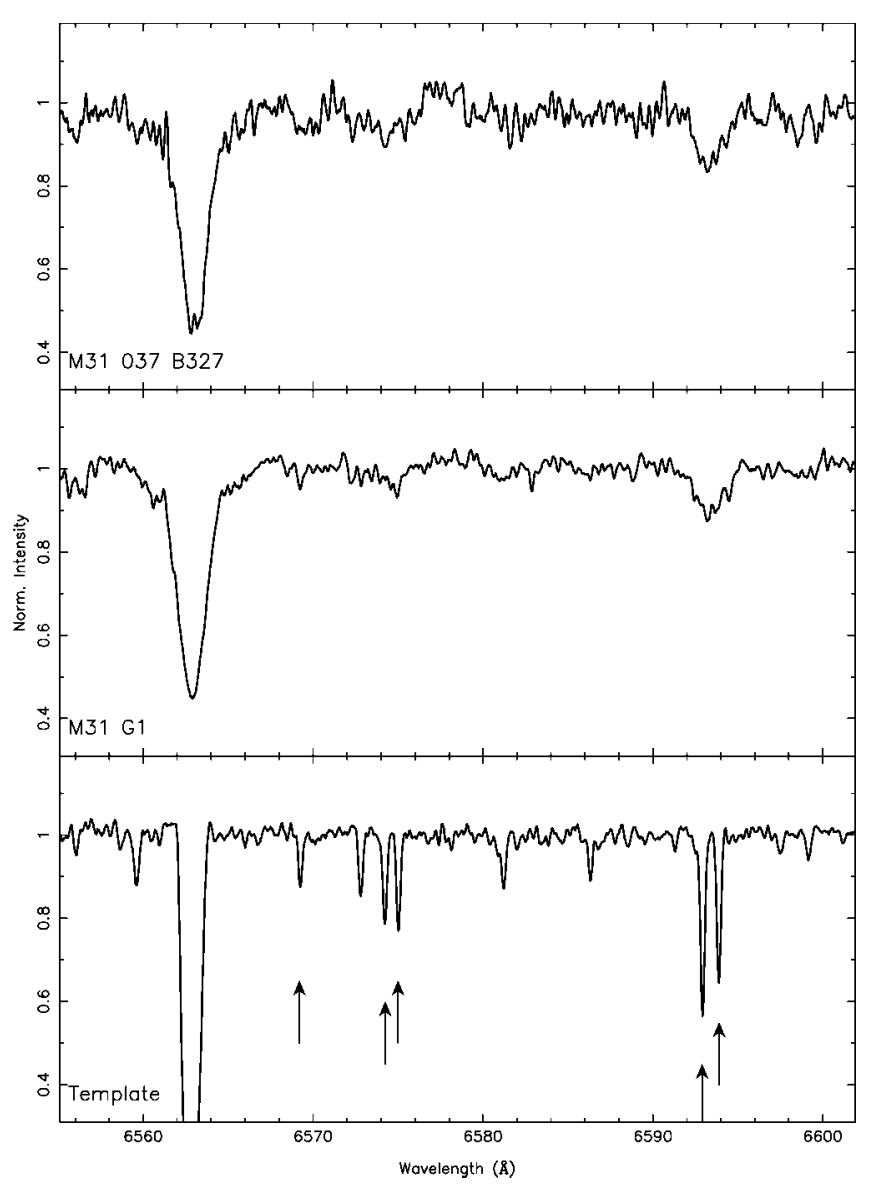

FIG. 1.-Region of the spectrum including $\mathrm{H} \alpha$, shown for 037-B327 (top), G1 (middle), and a template luminous red giant (bottom). The spectra have been normalized, shifted to the rest frame, and slightly smoothed by a Gaussian with FWHM $=5 \mathrm{~km} \mathrm{~s}^{-1}$. The spectral features marked by arrows in the bottom panel are all $\mathrm{Fe} \mathrm{I}$ lines.

(2006a), which suggested that $037-B 327$ is a factor of $\sim 2.5$ times more luminous than G1.

In late 2006 September we acquired an image at $K$ of the field of 037-B327 with the Wide-Field Infrared Camera (Wilson et al. 2003) at the Hale Telescope on Palomar Mountain to verify the IR photometry of this cluster. We find $K_{s}=$ $11.06 \pm 0.05 \mathrm{mag}$ in the Two Micron All Sky Survey (2MASS; Skrutskie et al. 2006; Cutri et al. 2003) system for an aperture $11^{\prime \prime}$ in diameter. This is within $0.01 \mathrm{mag}$ of that derived from the 2MASS database by Galleti et al. (2004). ${ }^{3} K_{s}$ for G1 from

\footnotetext{
${ }^{3}$ Table 2 of Galleti et al. (2004) presents IR magnitudes measured from 2MASS transformed into the Caltech system using the transformation equation of Carpenter (2001). We made the required inverse transformation for the comparison quoted above.
}

2MASS as reported by Galleti et al. (2004) is 0.025 mag fainter than that of $037-B 327$. If one takes $E(B-V)$ for the heavily reddened cluster $037-\mathrm{B} 327$ as $1.3 \mathrm{mag}$ with a more realistic error than that adopted by Ma et al. (2006a) given the spatially varying reddening, of $\pm 0.3 \mathrm{mag}$, then $037-\mathrm{B} 327$ has $M_{K}$ brighter by $0.16 \pm 0.03 \mathrm{mag}$. We thus find that $037-\mathrm{B} 327$ has $L_{K}$ comparable to that of $\mathrm{G} 1$ and at most $20 \%$ larger, even allowing for a generous uncertainty in $E(B-V) . M_{K}$ is well known to be a good measure of the total luminosity for old stellar systems; the dependence of $L_{\lambda}$ on $[\mathrm{Fe} / \mathrm{H}]$ is smaller at $2.4 \mu \mathrm{m}$ than at optical wavelengths.

In order to determine the virial mass of each of these objects, we must assume that the same initial mass function prevails in both of these clusters and combine $\sigma_{v}$ with the half-light radius determined from $H S T$ or other high spatial resolution imaging. Ma et al. (2006b) have measured $R_{h}$ for 037-B327, while there are two independent and discrepant determinations of $R_{h}$ for G1 (that of Meylen et al. 2001 and of Barmby et al. 2002a). $R_{h}$ for the highly reddened cluster $037-\mathrm{B} 327$ is not larger than that of G1. Thus, there is no evidence that the highly reddened globular cluster 037-B327 is substantially more massive than G1.

\section{CONCLUSIONS}

The luminous object 037-B327 believed to be a globular cluster in M31 is indeed a massive object that may or may not be the nucleus of an accreted galaxy, as was suggested by Meylan et al. (2001) and others. However, although it is among the most massive globular clusters in M31, at least four other globular clusters, G1, G78, G280, and G213, studied by Djorgovski et al. (1997) whose earlier $\sigma_{v}$ for G1 is confirmed here, have comparable masses. The first three of these are probably more massive than 037-B327. Among the Galactic globular clusters, $\omega$ Cen and NGC 6441 have $\sigma_{v}$ comparable to that of 037-B327, based on the compilation of Pryor \& Meylan (1993). Contrary to the conclusion of Ma et al. (2006a), we find it to be very similar to the well-studied cluster G1. The globular cluster 037-B327 is not an extraordinarily massive old cluster and is almost certainly not the most massive cluster of any age in the Local Group.

The entire Keck HIRES user community owes a huge debt to Jerry Nelson, Gerry Smith, Steve Vogt, and many other people who have worked to make the Keck Telescope and HIRES a reality and to operate and maintain the Keck Observatory. We are grateful to the W. M. Keck Foundation for the vision to fund the construction of the W. M. Keck Observatory. The authors wish to extend special thanks to those of Hawaiian ancestry on whose sacred mountain we are privileged to be guests. Without their generous hospitality, none of the obser-

TABLE 1

Velocity Dispersion Measurements for Two M31 Globular Clusters

\begin{tabular}{ccccc}
\hline \hline ID & $\begin{array}{c}\text { Wavelength Range } \\
\AA\end{array}$ & $\begin{array}{c}\sigma_{v} \\
\left(1 \sigma, \mathrm{km} \mathrm{s}^{-1}\right)\end{array}$ & $\begin{array}{c}\text { Uncertainty } \\
\left(\mathrm{km} \mathrm{s}^{-1}\right)\end{array}$ & $\begin{array}{c}\text { Apperture Correction } \sigma_{v}{ }^{a} \\
\left(\mathrm{~km} \mathrm{~s}^{-1}\right)\end{array}$ \\
\hline 037-B327 $\ldots \ldots .$. & $5150-5190$ & 19.2 & 3.5 & 21.9 \\
& $6105-6190$ & $\ldots{ }^{\mathrm{b}}$ & $\ldots$ & $\ldots$ \\
G1 $\ldots \ldots \ldots \ldots .$. & $6545-6600$ & 19.9 & 3.4 & 22.6 \\
& $5150-5190$ & 19.6 & 1.8 & 22.4 \\
& $6105-6190$ & 22.3 & 1.5 & 25.4 \\
& $6545-6600$ & 22.4 & 1.5 & 25.6 \\
\hline
\end{tabular}

${ }^{a}$ Aperture correction set to a factor of 1.14 following Djorgovski et al. (1997).

${ }^{\mathrm{b}}$ Features were too weak to permit a reliable determination of $\sigma_{v}$ in this spectral region. 
vations presented herein would have been possible. This publication makes use of data from the Two Micron All Sky Survey, which is a joint project of the University of Massachusetts and the Infrared Processing and Analysis Center, funded by the
National Aeronautics and Space Administration and the National Science Foundation. J. G. C. is grateful to NSF grant AST 05-07219 for partial support.

\section{REFERENCES}

Barmby, P., Holland, S., \& Huchra, J. P. 2002a, AJ, 123, 1937

Barmby, P., Huchra, J. P., Brodie, J. P., Forbes, D. A., Schroder, L. L., \& Grillmair, C. J. 2000, AJ, 119, 727

Barmby, P., Perrett, K. M., \& Bridges, T. J. 2002b, MNRAS, 329, 461

Baumgardt, H., Makino, J., Hut, P., McMillan, S., \& Portegies Zwart, S. 2003, ApJ, 589, L25

Carpenter, J. C. 2001, AJ, 121, 2851

Cutri, R. M., et al. 2003, Explanatory Supplement to the 2MASS All Sky Data Release (Pasadena: IPAC), http://www.ipac.caltech.edu/2mass/releases/allsky/ doc/explsup.html

Djorgovski, S. G., et al. 1997, ApJ, 474, L19

Galleti, S. F. L., Bellazzini, M., Fusi Pecci, F., \& Macrina, S. 2004, A\&A, 416, 917

Gebhardt, K., Rich, R. M., \& Ho, L. 2002, ApJ, 578, L41
Harris, W. 1996, AJ, 112, 1487

Ma, J., et al. 2006a, MNRAS, 368, 1443

. 2006b, ApJ, 636, L93

Meylan, G., Sarajedini, A., Jablonka, P., Djorgovski, S. G., Bridges, T., \& Rich, R. M. 2001, AJ, 122, 830

Pryor, C., \& Meylan, G. 1993, in ASP Conf. Ser. 50, Structure and Evolution of Globular Clusters, ed. S. Djorgovski \& G. Meylan (San Francisco: ASP), 357

Sargent, W. L. W., Schechter, P. L., Boksenberg, A., \& Shortridge, K. 1977, ApJ, 212, 326

Skrutskie, M. F., et al. 2006, AJ, 131, 1163

Vogt, S. E., et al. 1994, Proc. SPIE, 2198, 362

Wilson, W. C., et al. 2003, Proc. SPIE, 4841, 451 